\title{
The Differences of Resilience between Korean and Westerner: Concept Analysis
}

\author{
Hee Sook $\mathrm{Kim}^{1}$ and Wanju Park ${ }^{1 *}$ \\ Kyungpook National University and Research Institute of Nursing Science ${ }^{1}$ \\ wanjupark@knu.ac.kr
}

\begin{abstract}
Purpose: The concept of resilience is important to nursing for human adaptation. The aim of this paper is to enhance the understanding of resilience by clarifying the concept of Koreans' resilience by comparison with resilience of Westerners. Resilience is not yet static concept in Korea. After borrowing this concept from Westerners, it is still confused with ambiguous meaning in nursing. Methods: The technique developed by Walker and Avant was used as a guide in analyzing the concept of resilience in published literature. Results: Inner personal strength, intellectual ability, spirituality, and environmental protective factors were found to be the defining attributes of Koreans' resilience. Antecedents included pre-existing adversity and risk factors such as personal vulnerability, environmental risk, and lack of supportive resources. Consequences included adapting psychosocially, realizing one's ideals, protecting psychopathological problems, and contributing socially. This concept analysis can provide a fundamental definition of Korean resilience that is derived from Korean's unique 'Han' of cultural factors. Although Korean resilience is a totally new terminology in psychology, it has existed in Korean native consciousness and emotion. Conclusion: Korean's resilience is a more latent intrinsic capacity, which promotes adaptation despite the lack of social resources. Development of the ideal instrument for measurement of resilience of Koreans by defining definition, attributes, antecedents, and consequences identified from concept analysis with a focus on Korean culture and social background is needed.
\end{abstract}

Keywords: Concept analysis, Korea, Resilience

\section{Introduction}

Stressful circumstances or adversity are damaging to health and may lead to maladaptation. When under painful stress, we use different methods for management of this stress, according to patterns of culture and behavior. Koreans tend to view stress or adversity as an individual problem, not a social problem.

When working as an advanced psychiatric nurse in a Korea community, the researcher observed many different resilience phenomena, as some people, despite exposure to highly stressful circumstances, can withstand or bounce back psychologically without suffering psychological problems. However, this was different from resilience of Westerners. We can ask why some people are able to overcome adversity, whereas others succumb to life's hazards. When we borrow the concept of resilience from a different culture, there is a

\footnotetext{
* Corresponding Authors: Wanju Park

College of Nursing, Kyungpook National University and Research Institute of Nursing Science

101 Dongin-dong, Jung-gu, Daegu, Rep. of Korea, 700-422

Tel:+82-53-420-4977, FAX: +82-53-431-1020, E-mail: wanjupark@knu.ac.kr
} 
problem because of resilience is based on a diversity of socio-cultural influences. For this reason, each researchers use Korean's resilience in their own different ways.

The study of resilience is a fascinating subject. Since the late-1990s, resilience has been broadly understood as positive successful adaptation to circumstances involving extreme difficulties (Ki \& Chung, 2004; Kim, 1998; Lee \& Jo, 2005; Park, 1998; Yang \& Choi, 2001; Yoon, Hong, \& Lee, 2001). However, most of these studies borrowed the western concept of resilience; therefore, the term remains ambiguous.

Korean nurses wishing to use this concept may wonder at first about its true meaning. The meaning of resilience is difficult to pin down and the concept can be criticized for being too amorphous. The lack of consistency in definition of the concept, combined with the complexity of evaluation, has led to few conclusions regarding attributes and operational scale in resiliency. In order to use this concept in the nursing field, researchers are in dire need of consensus on the definition of Koreans' resilience, not Westerners' (Park \& Kim, 2014).

Walker and Avant (2005) stated that a concept analysis is useful in the effort to understand and refine a concept by reducing the ambiguity it is associated with, and is a fundamental process required by nurse-researchers who are attempting to measure the phenomena of nursing practices. Therefore, the finding of this study would provide many opportunities for development of nursing theory, as well as easy ways to use the concept of resilience in clinical nursing settings in Korea.

This paper is a revised and extended version of a brief article of the Park \& Kim's 2014.

\section{Method of Concept Analysis}

Walker and Avant's (2005) method for concept analysis was used in exploration of the concept of resilience. This study was conducted in order to clarify the meaning of a vague concept and to provide a precise operational definition of ego resilience through a review of nursing literature.

The technique developed by Walker and Avant (2005) was used as a guide in analyzing the concept of resilience. 1) Select the concept of resilience. 2) Determine the purpose of analysis. 3) Identify all uses and definitions of the concept. 4) Determine the attributes. 5) Identify concepts related to resilience. 6) Construct and identify cases. 7) Identify antecedents to the concept and consequences of the concept. 8) Define empirical references.

\section{Results of Concept Analysis}

\subsection{Step 1: Selection of the Concept}

The western concept of ego resilience was first introduced by Block in the mid-1970s (Park \& Kim, 2014). During the late 1990s, in Korea, researchers studying the concept of resilience wanted to know why some who are reared under different types of adversity appear to live healthy and productive lives while others seem never to overcome the adversity experienced during their lives.

Although the concept of resilience is useful, broadly, from specific children and adolescents to all age groups, the meaning and attributes of resilience have rarely been examined in Korea. The concept of resilience is not yet static in Korea. Therefore, many problems exist in using the concept of resilience, which was borrowed from different cultures without critical thinking. This lack of consensus on terminology and taxonomy of resilience in Korea indicates a need for more systemic analysis of this concept. Compared to the west, 
relatively little research on concept analysis of resilience in nursing has been conducted in Korea (Park \& Kim, 2014).

\subsection{Step 2: Purpose of Analysis}

In order to explore the meaning of resilience, and to provide a precise operational definition of resilience, a concept analysis of resilience is needed. Theoretical discussion of resilience is plentiful; however, the complex nature of resilience has resulted in little conclusive evidence regarding the process of resilience. When we attempted to use the Westerners' concept of resilience in Korean society, some inconsistencies or omissions were observed. Therefore, the conceptual analysis is conducted by comparison between Korea and the west. This study analyzed the concept of resilience through a review of literature in order to provide greater understanding of the definition and characteristics of its attributes (Park \& Kim, 2014).

\subsection{Step 3: Current Usage of the Concept}

3.3.1. Dictionary Definitions of Resilience: Dictionaries describe a concept in terms of its widely accepted and universal usage. Etymologically, the word 'resilience' comes from the Latin words 'salire' (to spring, spring up) and 'resilire' (spring back, leap back) (Davidson et al., 2005). Resilience is defined as flexibility in a situation and resistance to external power, the status of bouncing back to an original condition after a substance was bent or stretched (Yonsei Korean dictionary, 2002). The Korean language did not use the concept of resilience in psychological adaptation, whereas it has a similar meaning in a Korean native concept 'Han', which has endured throughout more than 5,000 years of Korean history (Park \& Kim, 2014).

Discussion of resilience in terms of human ability using the western concept indicates an ability to recover from or adjust easily to misfortune or change (The Merriam-Webster's Collegiate Dictionary, 2002). Other dictionaries define resilience as recovering, buoyant, bounce, elasticity, springiness, and flexibility.

3.3.2. Resilience Definitions of Literature Review: Considerable variations in the definition of resilience can be found in the Korean literature. Defining Korean resilience is difficult, because it is a borrowed concept. Therefore, although the meaning is the same, each researcher used different Korean words, including 'Tahl-Ruck-Sung' (flexibility), 'Juck-UngYoo-Yeon-Sung'(flexible adaptability), 'Hae-Bok-Ruck'(recuperative power), 'Kuck-BokRuck'(conquest, overcome), 'Bok-Won-Ruck'(restoring force, stability), 'Sim-Lee-JuckKeon-Kahng-Sung'(psychological health), etc. The Korean native concept, 'Han', has been regarded as a universal emotion of Korean people. 'Han' is a unique emotion, which refers to suffering experienced during a long and painful period of Korean history (Park \& Kim, 2014).

The positive attribute of 'Han' has induced motivation from a negative situation by alleviation and sublimation (Kim, 1991; Ko, 1988; Yang \& Choi, 2001). In the Korean language, 'Han' has been used for positive successful adaptation, because resilience means substance and is regarded as an economic term for elastic qualities (Park \& Kim, 2014).

Resilience combines several attributes from the research literature. Jung (1997) reported that resilience refers to individual traits of character. Resilience is an ordinary ability to cope effectively and with flexibility in response to external stress and inner tension (Yoon, Hong, \& Lee, 2001). Han (2008), Park (1998), Yoo and Shim (2002), and Lee and Jo (2005) reported on a number of protective factors and risk factors. Protective factors serve as buffers against negative effects of risk. Yoon et al., (2001) reported on the ability to change ego- 
control and cognition in stressful situations. Previous researchers have mainly reported that resilience of children and adolescents (Park, 1998), those who have run away from home (Han, 2008), and normal adolescents as well (Ku et al., 2001; Yoo \& Shim, 2002; Park \& Kim, 2014).

Most researchers have defined the resilience focus according to the style of Westerners and used the same measurement, which indicated uncertainty of the validity of the instrument for measurement of Korean resilience. Resilience is the ability to know how to use personal strengths, resources and energies to solve stressful situations (Klohnen, 1996; Connor \& Davison, 2003). However, Koreans do not usually try to use and access social resources when they fall into adversity (Park \& Kim, 2014).

3.3.3. Study Range and Stages of Resilience: From the late 1990s, pioneering researchers (Jung, 1997; Kim, 1998; Park, 1998) in Korea were psychologists and socialists interested in development of particular at risk children and adolescents. Previous work had focused on deficits and problems. Definition of resilience now ranges from simple to complex and there is a tendency to focus on all age groups (Masten, 2001). Resilience is regarded as an ordinary trait of basic human adaptation systems for extended all the ages. Resilience is not a set of fixed personal traits but instead a process of healthy human development. Current research has begun to view developmental phenomena in all phases of life, including mid-age and oldage (Staudinger, Marsiske, \& Baltes, 1993).

In addition, a pioneer Korean Researcher reported that resilience is a personal trait $(\mathrm{Ku}$, Hwang, \& Kim, 2001), that means resilience does not change during a lifetime. Many of the early theories on resilience focused on the role of genetics. It is now known that although genes and personality are important, they are influenced by many environmental factors, such as interpersonal support or available resources.

A resilience concept is now being used by many researchers in order to solve complex problems of human adaptation. Therefore, research on resilience has stimulated research in a wide variety of disciplines, including epidemiology, sociology, education, psychology, and psychiatry. Conduct of multi-disciplinary research may allow for integration of biological, psychological, and sociological perspectives on adaptation and development (Park \& Kim, 2014).

Resilience may be a useful concept not only when applied to individuals but also when applied to certain groups. Some social systems, including families (Ki \& Chung, 2004), schools (Park, 1998), and cultural groups or communities (Lee \& Jo, 2005) have been studied by nursing researchers. That means that the paradigm shift to a strengths model focuses on building an individual, a group, a family, or a set of community strengths. In future research, presentation of discussions in terms of specific domains of successful coping, such as academic resilience, social resilience, trauma resilience, or emotional resilience, would be more useful.

Most research on resilience focuses on personal traits and family domains of protective factors in Korea; therefore, they fail to recognize protective factors in a school or a community. Another problem is that Koreans do not use external support systems easily when experiencing adversity or painful stress. Compared to the Westerner, Korean people believe that such an issue is their personal problem; therefore, as a cultural custom, they do not open, share, or seek support systems or resources. In order to achieve an understanding of their resilience, identification of national characteristics, culture and race, social environment and connection or coherence of community, relation of family, and social support are should be included. In conclusion, deliberating on socio-cultural background is a requisite to application of resilience in a unique culture. 


\subsection{Step 4: Defining Attributes}

3.4.1. Internal protective factors: Inner personal protective factors can be seen as positive personal strengths, cognitive competency, and spirituality. Resilience is enhanced by an individual's internal-protective factors (Park \& Kim, 2014) (Table 1).

Personal characteristic strengths: 'Han' has a meaning of mitigation and sublimation (Yang \& Choi, 2001). It can endure and withstand adversity with constant intention to redirect in order to change. Sublimation of a strong desire can lead to socially acceptable development without failure or retaliation. Han is an emotion that is quite popular among Koreans; however, it could never be adequately translated into any western language. Neither could it be found with other Asian cultures, such as those of China or Japan (Park, 1999). The meaning of resilience has existed in Korean concept of 'Han' (Yang \& Choi, 2001; Park \& Kim, 2014).

Positive motivation: The positive motivation in the concept of 'Han' includes Korean's resilience. Positive personal strengths include strong self-esteem, self-efficacy, self-control, extroversion, honesty, responsibility, emotional stability, openness, autonomy, optimism, motivation, mastery-oriented, help-seeking behavior or a sense of humor (Park \& Kim, 2014).

Intellectual ability: Park (1999) reported that 'Han' is a perception and understanding of its entity and cause (Park \& Kim, 2014). Wright (1999) stated that an attempt to alleviate anguish indicates recognition of the existence of anguish. Control is the perception of oneself as having a definite influence through the exercise of imagination, knowledge, skill, and choice (Kobasa, 1979). It should include a cognitive system for acceptance of one's own adversity. Cognitive competency includes intelligence, IQ, attention skills, self-confidence, confronted risk, or problem solving skills (Park \& Kim, 2014).

Spirituality: Spirituality is a fundamental element of humanity and a natural internal need to pursue the meaning of life. Belief and spirituality exist in each experience that involves suffering. 'Shamanist performance of Han' of Korea is the process of mitigation and alleviation, followed by sublimation into adversity. 'Han' is a part of spirituality for the healing function. The 'Han' of Korean people might become a great spiritual energy (Park, 1999; Yang \& Choi, 2001; Park \& Kim, 2014). The endogenous factor of spiritual 'Han' as a coping process is proposed for resilience of Koreans. Spirituality is included in spiritual wellbeing, faith, belief, hope, vitality, tolerance, meaningfulness of life, sense of belonging, or connectedness (Park \& Kim, 2014).

3.4.2. External Protective Factors: External protective factors help to promote resilience. Resilience is enhanced by family, school, and community protective resources (Park \& Kim, 2014) (Table 1).

Family protective factors: A large family system and filial piety have an influence on resilience by family protective factors in Korea; however, due to importation of a western lifestyle such as a nuclear family, this has decreased. These family-protective factors include in at least one secure attachment relationship, good parenting, socioeconomic advantage, family cohesion, and lack of discord or tension in the family (Park \& Kim, 2014).

Community protective factors: External protective factors, such as social support or social resources, are insufficient in Korea. However, recent researches have suggested most important variable of resilience is how to use of available community resources or support systems like Westerner. School and community protective factors are included in positive school and community experiences, other opportunities to learn or qualify for advancement in 
society, teacher's support, teacher's good role, care of community facilities or churches (Park \& Kim, 2014).

\subsection{Step 5: Concepts Related of Resilience}

3.5.1. Ego Control: Individual differences in dimension of ego-control vary from overcontrol to undercontrol (Block, 2002). Persons with an internal locus of control demonstrate better overall adjustment than persons with an external locus of control (Block, 2002). Overcontrolled individuals tend to control or suppress impulse, needs, and feelings, whereas undercontrolled individuals allow their impulses free rein. Both overcontrollers and undercontrollers tend toward ego-brittleness, which is at the opposite end of the resilience continuum.

Ego-control refers to the tendency to contain rather than express impulses, desires, and emotions and can be represented as a continuum between overcontrol and undercontrol. Characteristically, undercontrolled individuals express impulses with relative immediacy and directness. They are unable to delay gratification, are self-dramatizing, unpredictable, have fluctuating emotions, and are easily distracted, and are relatively unbound by social customs. Overcontrolled individuals are inhibited in their actions and affect expressiveness to the point of being excessively constrained at times.

Conversely, resilient individuals have been described as having wide-ranging interests and a high level of aspiration, being assertive, socially poised, skilled, and cheerful. Resilience is not self-defeating, emotionally bland, nor lacking personal meaning in life (Block, 2002). Resilience is defined as the dynamic capacity of individuals to achieve appropriate modification of impulse control. Therefore, the internal locus of control is a protective factor of resilience.

3.5.2. Hardiness: The personality trait of 'hardiness' or 'toughness' helps to buffer extreme stress. Hardiness is an effective personal resistance-resource that can diminish potentially negative effects of life stress (Kobasa, 1979). In a stress-laden human environment, a hardy personality inherently adopts health-promoting factors (Kim, Kang, \& You, 2009). Therefore, hardiness is an important psychological health-promoting resource that can mediate an individual's responses in stressful situations.

Kobasa (1979) termed these situations as challenge, commitment, and control for hardiness. Hardiness is the composition of control, commitment, and challenge. Challenge is a belief that change rather than stability is normal in life. Hardy individuals view change as a challenge rather than a threat. Commitment refers to a generalized sense of purpose that allows individuals to identify with and find meaning in the events, things, and people in their environment. Hardy individuals believe in the importance and value of themselves and their experiences or activities. Hardy individuals believe that they can control or influence events. Kim, Kang, and You (2009) affirmed hardiness, which is an inherent part of personality, in contrast to resilience, and which is developed as a process.

In nursing, hardiness has also been studied in management, adaptation to chronic illness, family adaptation to stressors, and coping with war trauma. The partial functional equivalent of resilience is used in the sense of hardiness. Resilience is a higher level concept than hardiness. Resilience refers to the ability to adapt with flexibility and elasticity to changing circumstances.

3.5.3. 'Han' of Korean Native Concept: The native Korean concept of 'Han' is at first an emotional concept of enmity or self-reproach, is also an intrinsic phenomenon of motivation 
and a tendency to solve problems using indirect methods (Ko, 1988). 'Han' must be understood to originate from all painful experiences.

Korean's native consciousness and emotion has existed in the Korean concept of 'Han' that can be understood not on a pathologic perspective but on a normative and healthy one (Yang \& Choi, 2001). 'Han' is an emotion of desire and accomplishment for something in mind. The sentiment of 'Han' occurs when the long pains of suffering and trauma are combined with a person's strong desire and wishes regarding his or her life (Park, 1999). Lack of emotion, such as desire or achievement, can lead to rancor, or despair. Therefore, 'Han' is a latent ability to desire change (Chun, 1985; Ko, 1988; Yang \& Choi, 2001), Park (1999) reported that 'Han' does not conceal painful stress but participates in the actual life with a new vision for change. Kim (1991) reported that 'Han' is a dynamic psychiatric power involving the ability to change the future. Therefore, the Korean native concept of 'Han' is the ability to recover continually from stressful adversity and to have the intension of positive adaptation.

\subsection{Step 6: Variable Cases}

3.6.1. Model Case: Ms. Y. H. is 17 years old. When she was four years old, she suffered from poliomyelitis. She finally became physically disabled in her left leg with a limping gait. Her mother had suffered both physical and verbal abuse from husband who was an alcoholic. Her mother also suffers from major depression. She, however, is very sweet, trusting, and reliable to others. She cares for both parents with devotion. She graduated from high school with good academic achievement and has been working as a clerk at a small firm. Despite her physical disability, she is not only smart, confident, emotionally stable, open-minded to the world, but also has a high level of self-assurance. She challenges the future and has attended night school, having positive plans for the future, and delaying her gratification because she has 'Han' about her adversity. She has many close friends and close relationships with mentors in her church.

This example is an illustration of all of the defining attributes of the concept (Walker \& Avant, 2005). She exhibits the attributes of resilience. She desires to continue interaction with friends and community when she needs some help and is constantly positive in her selfassessment of her abilities and goals. Despite physical failure in her life, resilience is evidenced by her independence and autonomy.

3.6.2. Contrary Case: Mr. $\mathrm{S} . \mathrm{N}$ is 18 years old, and is now living with his father and a younger brother. His biological mother has been living alone in the next town. He knows that his mother has a boyfriend, as his father has a girlfriend. Although his mother occasionally gives him an allowance and helps him with meals, he still exhibits hostility toward his mother. He always lacks flexibility and adaptability in stressful situations and is afraid of changing circumstances. He is easily vulnerable, oversensitive, and upset about others. He always says "I am miserable. I can't do anything. Everything is impossible for me". His negative emotion, pessimism, and hostile feelings sometimes elicit aggressive behavior toward others. As a middle school student, he was arrested more than once for delinquency and violence, and was then expelled from high school. He was unemployed for at least one or two months, so that he just hung around without doing anything in particular.

This is a clear example of what the concept of resilience is not (Walker \& Avant, 2005). This is an example of a contrary case because he shows the opposite of attributes of resilience. He has isolated himself from family and community, and is depressed, with frequent mood 
swings. He no longer has any desire or willingness to accomplish anything. This type could be called a contrary case.

3.6.3. Borderline Case: Ms. J. E. is 19 years old and has been living with a two-year-old baby girl. She is a single teenage mother without relatives because she was an orphan. After separating from her boyfriend, she lived a more active lifestyle and demonstrated a more positive attitude in her job and in the community than she had done previously. She is always cheerful and has good relationships with her friends. She has a reputation for accomplishment of her duties in the grocery shop where she works. She is enthusiastic about hobbies, and about future plans. Everyone envies her passion and wants to become her close friend. However, she does not provide a good maternal role for her baby. Because she is busy, she neglects and abuses her daughter and leaves her unsupervised day and night. She hid this problem not even close to friends. She did not try to find solutions to her problems from any resources, including family, friends, or community.

Despite the presence of some defining attributes of resilience, this case lacked important attributes of resilience, including stable emotional openness, problem solving, and access to resources. This borderline case exemplified less than a model case. She is not willing to make any modifications to her lifestyle. She does not control her emotions and behavior toward her baby. She appears well-adapted from her divorce and continues to interact with co-workers; however, she does not accept the fact she is depressed and does not seek assistance or resources.

3.6.4. Related Cases: The first case involves an over-controlled ego. Mr. S. D. is a 19-yearold man who is very shy and timid. His mother ran away from her alcoholic husband when he was six years old. He is very dependent and socially isolated. He has never tried to drink or smoke because he has been afraid of following his father's behavior style. He has sometimes suffered loss of confidence and experienced extreme anxiety about losing control. As a consequence, he feared that he would become an alcoholic who indulges in ruinous habits. This is a case of so-called over-controlled ego.

The second case, a 14-year-old male whose mother had left home when he was seven years old, involves an under-controlled ego. Since his mother eloped and deserted him, he has experienced many adaptation problems including schoolwork, delinquency, and emotional problems, particularly external behaviors. This very self-centered man behaves impulsively and plays tricks, has outbursts of furious behavior, and shows antisocial patterns of behavior. Even though he is aggressive, his self-esteem is strongly sustained above a normal range and he cannot bear any delay of his needs. He was expelled from his middle school due to possession of illegal drugs.

The third case illustrates hardiness. Mr. S. S. is 13 years old, and living with a father who is an alcoholic. His mother ran away from her alcoholic husband when Mr. S. S. was a baby. In spite of his father's verbal abuse, he cares for him with patience. He only hopes that his difficult situation might help him in the future, so that he can bear everything and study hard under adversity. He has never complained about his situation, and has always hidden his story.

The first and second cases involve ego-control; that is, a concept related to resilience. Although these cases can demonstrate ego-control, the first case involved an over-controller and the second involved an under-controller. The third case illustrates individual hardiness only. He never tried to utilize any resources from family, school, or community. He did not use flexibility in coping with difficult situations; rather, he simply accepted and endured that situation alone. 


\subsection{Step 7: Antecedents and Consequences}

3.7.1. Antecedents: Antecedents are necessary conditions that must be present for resilience to matter (Walker \& Avant, 2005). Antecedents are applied to specific early predictors of such later outcomes. Stress is subjective experience and the personal interpretation. Inner personal risk factors increase symptoms of psychopathology or interrupt the ability to reach one's desired goals (Park \& Kim, 2014) (Table 1).

Pre-exist adversity: Pre-existing severe traumatic events include abuse, neglect, disruption, bullying, poverty, illness related resilience, or cumulative risk, etc.

Perceived and fall into stressful situation: Perceived stressful situations, such as adversity, are needed as antecedents. Adversity cannot be overcome by usual coping strategies, such as PTSD, neglect, poverty, trauma, abuse, or crisis situations.

Internal risk factors: Neurological disorders, difficulties with emotional regulation and expressiveness, aggression and difficulty in controlling impulses and behaviors, social incompetence and impaired or limited social relationships, antisocial personality and behaviors, other psychiatric dysfunctions and difficulties, rancor, or grief are inner personal risk factor.

External risk factors: Poverty, abuse, malfunction of family is environmental risk factor: A broken home, family trauma, family discord, parental dissension, repeated failure in school and at work, or stressors such as chaotic environments.

3.7.2. Consequences: Walker and Avant (1995) suggested that examination of consequences can shed significant light on the social and cultural context in which the concept is generally used. Consequences are the results or outcomes of resilience. The nature of desirable or undesirable developmental outcomes is shown as follows (Table 1):

Positive consequence: Chun (1985) reported that the person who has experienced 'Han' has empathy, plentiful susceptibility, and acceptance of another person's suffering. 'Han' is a motive power of the realization of one's hopes, growth, and development of the individual by the sublimation of resilience. This involves individual growth and development as well as social contributions across social contexts. Therefore, positive consequences include psychosocial adaptation, individual development, self-actualization, protection of psychopathological problems, mental and spiritual health and well-being, strength-focused, or social contributions across social contexts (Park \& Kim, 2014).

Negative consequence: When 'Han' remains in rancor, lamentation, and malice, negative consequences, such as anxiety, depression, and high risk behavior problems, occur. Therefore, negative consequences include psycho-social mal-adaptation, high-risk behavior problems, social withdrawal, fixed developmental stages, and mental emotional problems, or psychopathological problems (Park \& Kim, 2014).

\subsection{Step 8: Empirical References}

According to Walker and Avant (2005), empirical references are defined as the way in which a concept can be measured, that there are classes or categories of actual phenomena, which, by their existence, demonstrate occurrence of the concept. In order to be clear about the importance of resilience, the concept must be operationally defined.

The Ego-Resiliency Scale (ERS) of the Korean Personality Inventory for Children (KPI-C), conducted by Kim et al., (1997), included the capacity for measurement of peer relations and self-esteem, self-acceptance and optimism, and family relations. Ju and Lee (2007) developed the Resilience Scale for Children (RSC), which consists of 30 items and five subscales that 
include self-efficacy, tolerance of negative affect, positive support relations, power of control, and spontaneity.

Understanding of preschool children is difficult because they do not express themselves with accuracy and objectivity with regard to their level of resilience; therefore, the parent form of the resilience scale was constructed by Yoon, Hong, and Lee (2001). Items included in the scale are selected from the Korea Personality Inventory for Children (KPI-C) Preliminary Form. Five factors, including peer-relationship and optimism, empathy and selfacceptance, attention and self-esteem, comprehension, and leadership are extracted. In development of the resilience scale for Korean adolescents, Lee and Jo (2005) developed a resilience scale containing 48 items of 16 resilience factors for Korean adolescents.

Many foreign instruments are used in Korea. Klohnen (1996) developed a 29-item selfreport scale of ego-resilience. Use of this measure may result in determination of ego resilience (ER). Wagnild and Young's (1993) Resilience Scale (RS) was developed from a study conducted among elderly women. This scale is a 25 -item self-report questionnaire, a 7point Likert response scale for evaluation of personal competence, acceptance of self and life, and balance and flexibility. The Resilience in Midlife Scale (RIM) is a 25-item for measurement of dimensions of five factors, including self efficacy, family and community network, coping and adaptation, tolerance, and control (Ryan \& Caltabiano, 2009). The 25item Connor-Davidson Resilience Scale (CD_RISC) was developed for measurement of dimensions of five factors in various age groups: personal competence, affect tolerance, acceptance of change, sense of internal control, and spirituality for measurement of PTSD (Connor \& Davison, 2003). The Resilience Scale for Adults (RSA) contains 37 items and five subscales, which include personal competence, social competence, family coherence, social support, and personal structure (Friborg, Hjemdal, Rosenvinge, \& Martinussen, 2003) (Table 2).

Resilience should be measured in terms of both internalizing and externalizing risk, protective factors, antecedents, and consequences. In addition, researchers should consider cumulative risks and adversity that often accumulate in our lives, rather than a single event. And, measurement of the developmental level of individuals or the duration or severity of disruption has not often been undertaken. Most instruments of resilience measure only protective factors. Only few scales have been developed for measurement of environmental protective factors, antecedents, and consequences. A multi-dimensional approach to research is also needed. Empirical references show a lack of consensus with regard to the optimal method for measurement of attributes of resilience. There is a need to distinguish between chronic and acute stressors, uncontrollable and controllable risks, desirable and undesirable outcomes, and proximal and distal factors too. Resilience, as a concept, appears to cross national and cultural boundaries. Therefore, the lack of rigorous empirical analysis makes it difficult to use the concept in nursing.

\section{Discussion}

The purpose of this study is to analyze the concept of resilience through a review of nursing literature and to provide a clearer understanding of the perspective of the concept of resilience. The technique developed by Walker and Avant (2005) is used as a guide in analysis of the concept of resilience.

Although resilience is not used in psychological aspects in Korea, there is a similar meaning in Korean awareness, emotion, and culture. That concept of resilience exists in the native Korean concept of 'Han', which affects Koreans' behavior or Koreans' way of thinking. The concept of 'Han' has a positive meaning, which is closely connected to 
successful adaptation and change. Korean resilience remains associated with individual attributes. If external protective factors were offered, the process of development and improvement of resilience would be easier.

The concept of resilience is multidimensional and complex. Thus, resilience is a term that has been used by different researchers in a variety of different ways. By combining the attributes derived from literature on Korean resilience, a definition of resilience has been developed. Resilience is a complex construct and a universal human capacity to face a latent psychosocial capacity which promotes adaptation under adversity across social contexts.

This concept analysis of resilience examines all of the factors of attributes that contribute to adaptation by intrinsic protective factors and extrinsic protective factors, such as inner personal strength, intellectual ability, social skills and spirituality, environmental protective factors, and easy access to protective factors and resources.

In addition, a precipitated event, such as trauma or adversity, a perceived stressful situation with regard to that event, and risk factors that are individual risk factors or environmental risk factors were identified as antecedents to resilience. Positive consequences of ego resilience include psychosocial adaptation, individual growth and development, realization of one's ideals, protective psychopathology problems, mental and spiritual health and well-being, and capacity for social contributions across social contexts. Negative consequences include psychosocial maladaptation, high risk behavior problems, social withdrawal, developmental fixation, and mental or emotional problems. Resilience is focused on positive aspects of human ability; therefore, the concept of resilience allows for the potentiality of developing tools to encourage recovery from disease and promotion of health through nursing.

The definition and operational method presented here can be used by health professionals in development of individual, family, and community programs that promote resilience. Foreign instruments developed in different social-cultural backgrounds are not familiar with regard to questionnaire or terminology, and should be examined according to the validity of the instrument as used in a Korean social-cultural situation (Yoo \& Shim, 2002).

In conclusion, Korean resilience focuses on individual personal strength for adaptation by use of sublimation of 'Han' whereas Westerners' resilience includes more extended resources or social support for development of a positive direction (Figure 1).

\section{Conclusion}

Resilience is a continuous critical concept for use in investigations of how resilience is less determined by the stressors experienced and more related to resources available to address the stressors, how people deal with life changes and the manner in which they address their situation during the process of change. Further studies are needed for reevaluation of the result of this study, suggesting that 'Han' is related to resilience of Koreans. This concept analysis study of Korean resilience has enabled nurses to understand which factors place humans' adaptive development in jeopardy and to offer information on how best to assist clients who need to increase resilience for achievement of successful adaptation (Park \& Kim, 2014).

Korean instruments should be developed and should include new strategies, such as a resource-based approach, social environment, in order to bolster insufficient social support and resources as external protective factors. Risk and protective factors of resilience differ significantly according to a unique cultural tendency. Resilience is affected by risk factors and protective factors; therefore, due to differences of culture, language, and customs, each country has its own uniquely defined concept of resilience and unique instruments. In 
addition, nursing intervention to encourage resilience should be tailored to culturally specific solutions (Park \& Kim, 2014).

Table 1. Antecedents, Attributes, and Consequences of Korean's Resilience

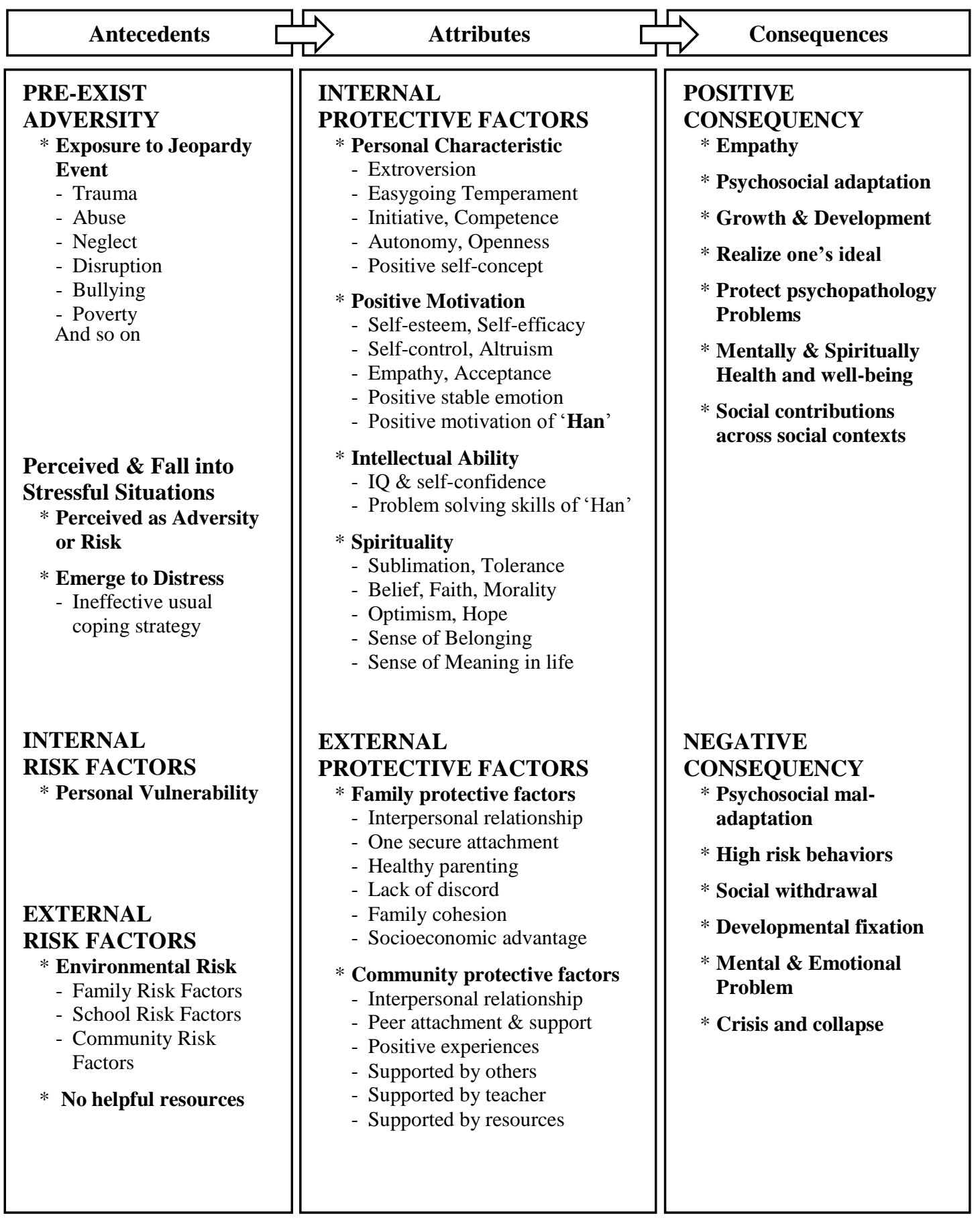


Table 2. Empirical References for Resilience in Resilience of Westerners and Koreans

\begin{tabular}{ll}
\hline Instruments Name of Resilience & Developer of Instrument \\
\hline Ego Resilience Scale (ER) & Klohnen (1996) \\
Ego Resilience Scale (ER89) & Block \& Kremen (1996) \\
Resilience Scale (RS) & Wagnild \& Young (1993) \\
Connor-Davidson Resilience Scale (CD_RISC) & Connor \& Davision (2003) \\
Resilience Scale for Adults (RSA) & Friborg et al. (2003) \\
Resilience in Midlife Scale (RIM) & Ryan \& Caltabiano (2009) \\
\hline Korean Ego-Resiliency Scale (ERS) & Kim et al. (1997) \\
Korean Parent Form Ego-Resilience Scale & Yoon et al. (2001) \\
Resilience Scale in Korean Adolescents & Lee \& Jo (2005) \\
Resilience Scale of Children (RSC) & Ju \& Lee (2007) \\
\hline
\end{tabular}

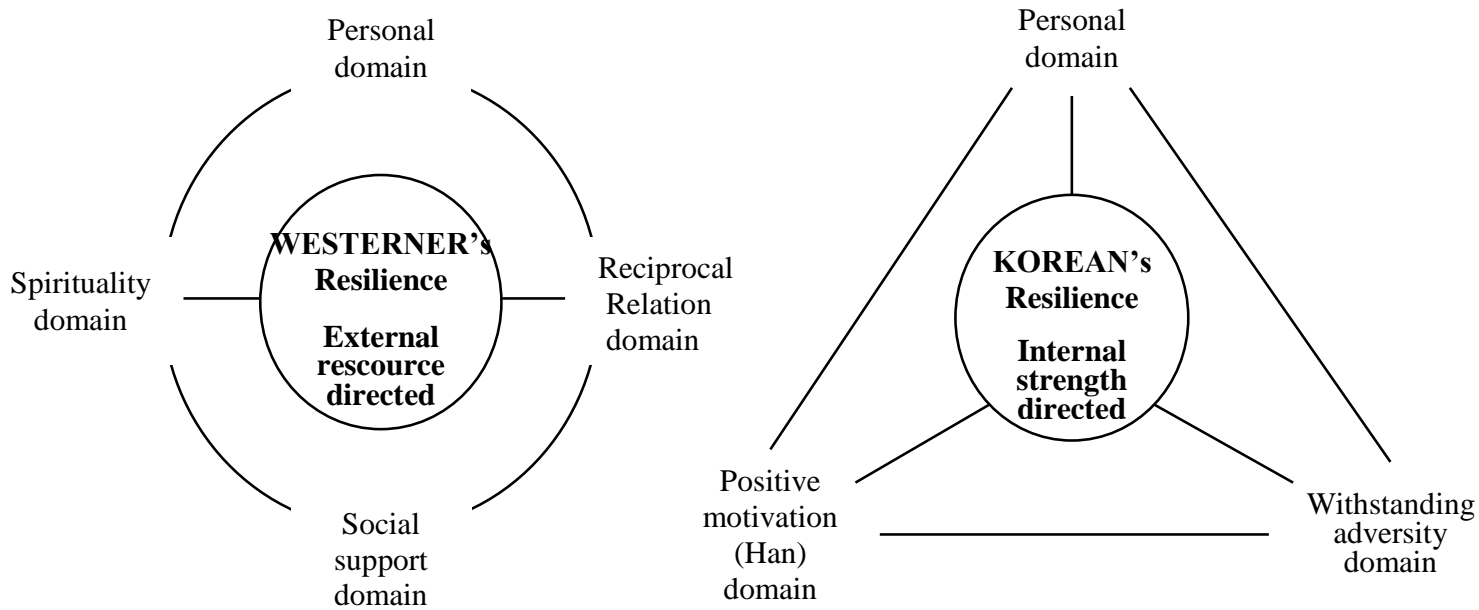

Figure 1. Comparison of Resilience between Westerner and Korean

\section{Acknowledgements}

Special thanks should be given to Dr. Haeok Lee, my research supervisor, for her professional guidance and valuable support and constructive recommendations during the planning and development of this research work. I would also like to extend my thanks Dr. Kim, co-author and colleague, for sharing many ideas in offering me the resources in running the concept analysis.

\section{References}

[1] J. Block, "Personality as an affect-processing system", Erlbaum, Mahwah, (2002).

[2] K. M. Connor and J. R. Davison, "Development of a new resilience scale: The Connor-Davison resilience Scale (CD-RISC)", Depression and Anxiety, vol. 18, no. 2, (2003), pp. 76-82.

[3] E. D. Chun, Korean literature and Han. E-Yoo Publish Co., Seoul (1985).

[4] J. R. T. Davidson, V. M. Payne, K. M. Connor, E. B. Foa, B. O. Rothbaum, M. A. Hertzberg and R. H. Weisler, "Trauma, resilience, and saliostasis: Effects of treatment in post-traumatic stress disorder", International Clinical Psychopharmacology, vol. 20, (2005), pp. 43-48. 
[5] O. Friborg, O. Hjemdal, J. Rosenvinge and M. Martinussen, "A new rating scale for adult resilience: What are the central prospective resources behind healthy adjustment?", Journal of Methods in Psychiatric Research, vol. 12, no. 2, (2003) pp. 65-76.

[6] S. C. Han, "Exploration on risk and protective factors of adolescent runaway", Korean Journal of Psychological and Social Issues, vol. 14, (2008), pp. 273-298.

[7] D. H. Jung, "Resilience on stress in childhood and adolescence", The Journal of Research in Education, vol. 9, (1997), pp. 205-223.

[8] S. Y. Ju, and Y. H. Lee, "Development and validation of the resilience scale for children in grade 4 through 6 (RSC)", Journal of Child Studies, vol. 28, (2007), pp. 91-113.

[9] H. Ki, and N. W. Chung, "Resiliency model in families of children with chronic disease: Focusing on the psychological adaptation process", The Korean Journal of Psychology, vol. 9, no. 1, (2004), pp. 1-24.

[10] S. T. Kim, J. H. Kim, D. H. Song, H. K. Lee, Y. H. Joo, and C. H. Hong, KPIC, Korean Guidance, Seoul (1997).

[11] H. S. Kim, "Concept development of resilience", Journal of Nurses Academic Society, vol. 28, (1998), pp. 403-413.

[12] J. H. Kim, T. Y. Kang, and J. M. You, "Development of stress hardness scale", The Korean Journal of Health Psychology, vol. 14, (2009), pp. 175-191.

[13] Y. A. Kim, Han: Brokeness to wholeness, A dissertation presented to the faculty of the school of theology at Claremont (1991).

[14] E. C. Klohnen, "Conceptual analysis and measurement of the construct of ego-resiliency", Journal of Personality and Social Psychology, vol. 70, (1996), pp. 1067-1079.

[15] E. Ko, For conquer of Han. In Seo, G. P. (Ed), Story of Han, Publishing Co., Seoul (1988).

[16] S. C. Kobasa, "Stressful life events, personality and health: An inquiry into hardiness", Journal of Personality and Social Psychology, vol. 37, no. 1, (1979), pp. 1-11.

[17] H. M. Ku, S. T. Hwang, and J. H. Kim, "The personality traits of ego-resilience", The Korean Journal of Clinical Psychology, vol. 20, (2001), pp. 569-581.

[18] H. Lee, and H. I. Jo, "A study for development of the resilience scale of Korean adolescents", Studies on Korean Youth, vol. 16, (2005), pp. 161-206.

[19] A. S. Masten, "Ordinary magic: Resilience process in development", American Psychologist, vol. 56, (2001), pp. 277-238.

[20] Meriam-Webster Dictionary of the English Language (4th ed.) Houghton Mifflin, Boston (2002).

[21] J. S. Park, Han and Shinmyong, "The suffering soul and liberating life of the oppressed people", Korean Journal of Systematic Theology, vol. 3, (1999), pp. 195-224.

[22] H. S. Park, T'he developmental mechanism of school resilience of Korean adolescents in poverty", Korean Journal of Youth Studies, vol. 5, (1998), pp. 147-165.

[23] W. Park and H. S. Kim, "Korean's resilience compare to the Westerner: Concept analysis", Advanced Science and Technology Letters, vol. 47, (2014), pp. 323-327.

[24] L. Ryan, and M. L. Caltabiano, "Development of a new resilience scale: The resilience in midlife scale (RIM Scale)", Asian Social Science, vol. 5, no. 11, (2009), pp. 39-51.

[25] U. M. Staudinger, M. Marsiske, and P. B. Baltes, "Resilience and levels of reserve capacity in later adulthood: Perspectives from life-span theory”, Development and [1] Psychopathology, vol. 5, pp. 541-566, (1993).

[26] G. M. Wagnile, and H. M. Young, "Development and psychometric evaluation of the resilience scale", Journal of Nursing Measurement, vol. 1, (1993), pp. 165-178.

[27] L. Walker, and K. Avant, "Strategies for theory construction in nursing (4th ed.)", Pearson Prentice Hall, Upper Saddle River, NJ (2005).

[28] M. L. Wright, "Spirituality, suffering, and belief: The soul of healing with families", In spiritual resources in family therapy. edited by Walsh. F. Guilford, New York (1999).

[29] O. K. Yang, and M. M. E. Choi, "Korean's Han and resilience: Application to mental health social work", Psychological Science, vol. 11, no. 6, (2001), pp. 7-29.

[30] S. K. Yoo, and H. W. Shim, "Psychological protective factors in resilient adolescents in Korea", The Korean Journal of Educational Psychology, vol. 16, (2002), pp. 189-206.

[31] H. H. Yoon, C. H. Hong, and Lee, J. H. Lee, "Development of parent form ego-resilience scale", Journal of Psychological Science, vol. 10, (2001), pp. 33-53.

[32] Yonsei Korean dictionary, Doosandonga Co., Seoul, (2002). 